\title{
Inflammatory and Hyperproliferative Skin Disease in Mice That Express Elevated Levels of the IL-1 Receptor (Type I) on Epidermal Keratinocytes \\ Evidence That IL-1-inducible Secondary Cytokines Produced by Keratinocytes In Vivo Can Cause Skin Disease
}

\author{
Richard W. Groves, Tamara Rauschmayr, Koichiro Nakamura, Saumyendra Sarkar, Ifor R. Williams, and Thomas S. Kupper \\ Harvard Skin Disease Research Center, Brigham and Women's Hospital, Boston, Massachusetts 02115
}

\begin{abstract}
Interleukin (IL)-1 induces a cascade of secondary cytokines in a large number of cell types in vitro, including monocytes, fibroblasts, synovial cells, and keratinocytes. Although it has been proposed that autocrine or paracrine activation of such cells by IL-1 in situ could orchestrate a local inflammatory response, formal proof for such an hypothesis has been lacking. In an attempt to lower the threshold for secondary cytokine production in these cells in response to IL-1, we have generated transgenic mice (designated IR10) which overexpress functional type $1 \mathrm{IL}-1$ receptor in basal layer of epidermis keratinocytes. As predicted, keratinocytes from these animals were substantially more responsive to exogenous IL-1 than nontransgenic keratinocytes when stimulated in vitro. When challenged with known inducers of keratinocyte IL-1 synthesis and release, skin of IR10 mice exhibited an exaggerated inflammatory response, characterized by epidermal hyperplasia and an acute dermal inflammatory cell infiltrate. In this setting, the secondary epidermal cytokines gro- $\alpha$ and GM-CSF were strongly induced in transgenic epidermis but not in control skin. To confirm that these changes were indeed related to IL-1 mediated activation pathways, IR10 mice were crossed to a distinct line of transgenic mice that overexpress $17-\mathrm{kD}$ IL-1 $\alpha$ in basal keratinocytes. Double transgenic mice derived from this cross breeding experiment developed spontaneous inflammation of the skin, similar in appearance to that induced by PMA, both histologically and macroscopically, and distinct from that seen in either parental strain spontaneously. Furthermore, secondary cytokines were more strongly induced in the double transgenic than in either parental strain. These findings conclusively demonstrate the potential for functional autocrine pathways of keratinocyte activation mediated by IL-1 $\alpha$ in vivo, and suggest that level of expression of type $1 \mathrm{IL}-1$ receptor may function as a significant control point in physiologic IL-1 mediated autocrine pathways. (J. Clin. Invest. 1996. 98:336-344.) Key words: interleukin $1 \cdot$ cytokine $\cdot$ receptors $\cdot$ inflammation $\cdot$ skin
\end{abstract}

Address correspondence to T.S. Kupper, M.D., Harvard Skin Disease Research Center, Division of Dermatology, Brigham and Women's Hospital, 75 Francis Street, Boston, MA 02115. Phone: 617-278-0993. FAX: 617-278-0305; E-mail: tskupper@bics.bwh.harvard.edu

Received for publication 23 February 1996 and accepted in revised form 24 April 1996.

J. Clin. Invest.

(C) The American Society for Clinical Investigation, Inc.

0021-9738/96/07/0336/09 \$2.00

Volume 98, Number 2, July 1996, 336-344

\section{Introduction}

The cutaneous interleukin-1 (IL-1) axis is one of the most complex systems of cytokine agonists, antagonists, and receptors in cytokine biology $(1,2)$. Normal epidermis contains, in biological terms, large amounts of preformed and biologically active IL- $1 \alpha(3,4)$ in addition to significant quantities of immature IL-1 $\beta$ (5). Neither protein has a conventional signal peptide (6) and they therefore remain largely intracellular unless cell damage occurs, whereupon they are thought to be liberated into the extracellular space. The authentic in vivo relevance of this intracellular "store" of IL-1 activity has remained elusive, but one potential role is that of an immediate initiator of inflammatory and repair processes following injury to the integument $(1,2)$. IL-1 is well suited to this role because of its pleiotropic actions, including the activation of endothelium and the induction of multiple secondary cytokines that act upon inflammatory leukocytes (7).

Keratinocytes not only produce biologically active IL-1, but also respond to IL-1 in a number of ways that include the elaboration of several secondary cytokines such as IL-6 and GM-CSF (8). For these reasons, IL-1 has been termed a "primary" cutaneous cytokine $(1,2)$. These cellular responses to IL-1 are mediated through the keratinocyte type-1 IL-1 receptor $(6,9)$ that is typically expressed constitutively at low levels on the cell surface (10).

Presumably because IL-1 release has such profound inflammatory consequences, keratinocytes have developed a number of means of controlling its activity. The first of these to be identified was a specific epithelial form of the IL-1 receptor antagonist (IL-1ra) (15-17). This represents a variant of the secreted monocyte form of IL-1ra which, by virtue of alternate splicing, lacks a signal peptide and therefore, like IL- $1 \alpha$ and IL-1 $\beta$, remains entirely cell associated and is likely released with them after cell damage. A second intracellular form of IL1ra was recently identified in keratinocytes (18). Furthermore, both human and murine keratinocytes express the type-2, nonsignal transducing, IL-1 receptor (10-12). The precise role of this molecule is uncertain, but it is likely that by virtue of its lack of signal transducing ability together with high affinity binding of IL- $1 \alpha$ and IL-1 $\beta$ (but not IL-1ra), it functions as a further antagonist of IL-1 activity (13, 14, and Rauschmayr et al., manuscript submitted for publication). Together, the IL1ra and the type 2 IL-1R have the potential to effectively antagonize IL-1 mediated inflammatory responses.

Because keratinocytes both produce and respond to IL-1, and have evolved multiple ways of controlling its activity, we have previously postulated that highly regulated autocrine pathways centered on IL-1 exist in epidermis. Specifically, we have proposed that keratinocytes stimulated by the agonist activity of IL-1 will produce a series of secondary cytokines in 
vivo that should gain access to the dermis and influence the inflammatory response, both qualitatively and quantitatively. Though such ideas are bolstered by circumstantial evidence, formal proof of this hypothesis is lacking. To further study this question we have generated transgenic mice using the human keratin 14 promoter to target overexpression of functional type 1 IL-1 receptor to basal keratinocytes. In this study, we report that these mice exhibit exaggerated inflammatory responses to locally produced keratinocyte IL-1 that demonstrably modify the character of cutaneous inflammation in vivo.

\section{Methods}

Generation of transgenic animals. Type $1 \mathrm{IL}-1 \mathrm{R}$ cDNA (the kind gift of Dr. J. Sims, Immunex Research and Development Corporation, Seattle, WA) was excised from plasmid Gembl78 (19) with EcoRI and $\mathrm{XbaI}$ (Boehringer Mannheim, Indianapolis, IN), and cloned into the BamHI site of the $\mathrm{K} 14 / \mathrm{hGH}$ expression vector (20). The resulting construct was excised from the vector with EcoRI, gel purified and used for pronuclear injection of one-cell fertilized embryos of $\mathrm{FVB} / \mathrm{N}$ mice. The following day, two-cell embryos were transferred to pseudopregnant Swiss Webster recipients and the resulting litters were screened for incorporation of transgene by PCR analysis of ear skin DNA or by Southern blot analysis of tail DNA. Two founder animals were identified, designated IR3 and IR10, and were bred to establish lines heterozygous for the transgene. Mice used in the experiments described were 6-16 wk of age. Animals of the TgIL-1.2 line (21), which overexpress $17-\mathrm{kD}$ IL-1a in basal keratinocytes, were crossed to IR10 mice for certain experiments; mice which co-expressed both transgenes were identified by PCR analysis using primer pairs specific for each transgene.

RNA analysis. RNA was extracted from multiple tissues of transgenic and nontransgenic animals by homogenization in guanidinium isothiocyanate followed by centrifugation through a $5.7 \mathrm{M}$ cesium chloride cushion. $20 \mu \mathrm{g}$ total RNA was then fractionated on a $1 \%$ agarose/formaldehyde gel, blotted to nylon membrane (Hybond $\mathrm{N}+$, Amersham International) and hybridized to ${ }^{32} \mathrm{P}$-labeled probes generated by random priming (Promega Corp., Madison, WI). Probes used were as follows: a 450-bp BamHI fragment from the type 1 IL-1 receptor transgene; a 590-bp HindIII/DraI fragment of the murine gro- $\alpha$ gene (kind gift of Dr. C. Stiles, Dana Farber Cancer Institute, Boston, MA); a 530-bp AvaII fragment from the coding region of murine GM-CSF (the kind gift of Dr. F. Lee, DNAX Corp.) and a 501-bp cDNA encoding mature murine IL-1 $\alpha$ (R\&D Systems, Minneapolis, $\mathrm{MN})$.

Keratinocyte culture and GM-CSF ELISA. Keratinocytes were derived from adult mouse ears using sequential dispase and trypsin digestion (22). Primary cultures were established, split after two days into wells of a 96-well plate and then grown for $24 \mathrm{~h}$ in medium containing various concentrations of recombinant murine IL- $1 \alpha$ (kind gift of Dr. P. Lomedico, Hoffman LaRoche, Nutley, NJ). Medium contained $0.06 \mathrm{mM} \mathrm{Ca}^{2+}$ so that keratinocytes maintained an undifferentiated proliferative phenotype with persistent expression of keratin 14 (22). Immunoreactive GM-CSF in supernatants was assayed by ELISA according to the manufacturer's instructions (Genzyme Corporation, Cambridge, MA). Because of the possibility that normal and transgenic keratinocytes might not grow at the same rate, cells were removed from additional wells by brief trypsinization and counted to allow normalization of GM-CSF levels.

Flow cytometry. Epidermal cell suspensions were derived from adult mouse ears by sequential dispase and trypsin digestion. Cells were stained with antibodies against mouse type-1 IL-1 receptor (M15, rat IgG2a: gift of Dr. J. Sims, Immunex Research and Development Corporation, Seattle, WA; or 35F5, rat IgG1: gift of Dr. R. Chizzonite, Hoffman LaRoche, Nutley, NJ). Biotinylated anti-rat immunoglobulin and streptavidin-phycoerythrin second and third layer reagents were from PharMingen and Caltag Laboratories respectively. Stained cells were analyzed on a FACScan ${ }^{\circledR}$ flow cytometer (Becton Dickinson, Mountain View, CA) equipped with CellQuest software, and non-viable cells were gated out with propidium iodide before analysis.

In vivo PMA stimulation. Groups of 3-5 mice underwent application of $0.5 \mu \mathrm{g}$ phorbol 12-myristate 13-acetate (PMA; Sigma Chemical Co., St. Louis, MO) in $10 \mu \mathrm{l} 100 \%$ ethanol to each side of the right ear at the times noted. Ethanol alone was applied to the left ear as control. Ear thickness was measured by micrometer prior to the first applications and periodically thereafter.

Histology and immunohistochemistry. Tissue for histological evaluation was fixed in formaldehyde and embedded in paraffin. 5- $\mu \mathrm{m}$ sections were cut and stained with hematoxylin and eosin by conventional methods.

\section{Results}

Generation of transgenic animals. After pronuclear injection of the construct described above, two transgenic founders were identified by Southern blot analysis. These mice were bred to establish heterozygous lines, designated IR3 and IR10, which were used for all experiments. Animals from both lines bred normally and produced the expected Mendelian ratio of transgenic to nontransgenic offspring.

Tissue-specific expression of transgene $m R N A$ and protein. RNA blots of ear skin from IR10 and IR3 mice demonstrated high level expression of $\sim 4.5 \mathrm{~kb}$ transgene mRNA. The putatively low constitutive expression of $\sim 5 \mathrm{~kb}$ wild-type mRNA in nontransgenic animals could not be detected in control mice

28 S -

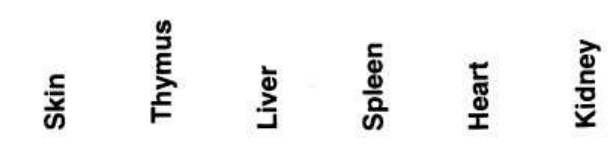

$18 \mathrm{~S}$ -

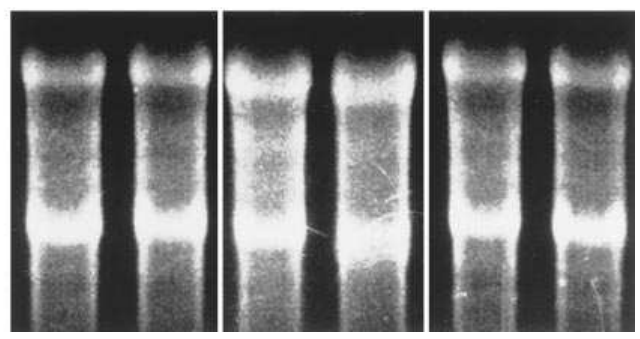

Figure 1. Tissue specificity of transgene expression in IR10 mice. (Upper panel) $20 \mu \mathrm{g}$ total RNA from various organs was fractionated on a $1 \%$ agarose formaldehyde gel as described, transferred to nylon membrane and probed with a murine type 1 IL-1 receptor cDNA. High level expression of transgene is seen in skin but not in other tissues. (Lower panel) ethidium bromide stained gel demonstrates equivalent RNA loading. 
FVB

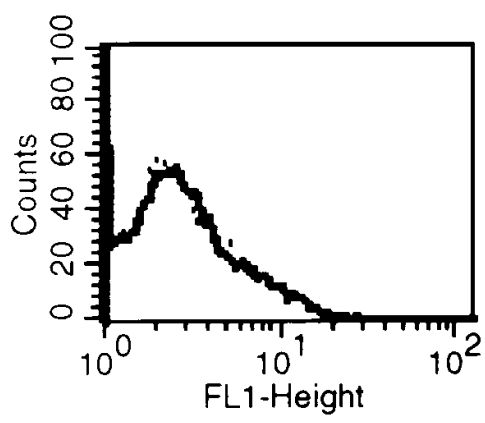

IR3

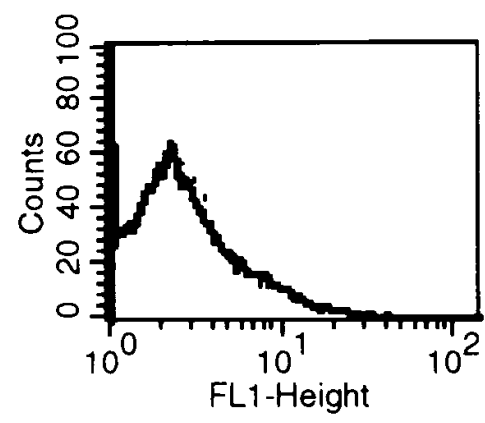

IR10

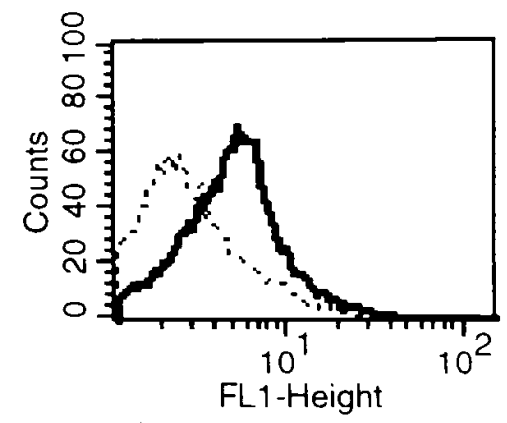

\section{IL-1R type 1}

Figure 2. Flow cytometric analysis of type-1 IL-1 receptor expression in keratinocytes derived from nontransgenic (FVB), IR3 and IR10 mice. Solid line, 35F5; dotted line, isotype control. There is high level expression of the transgene product on keratinocytes derived from IR10 mice but little or no detectable staining in IR3 and control animal keratinocytes.

(not shown). Densitometric analysis suggested that levels of mRNA expression in IR10 mice were at least 3-fold higher than in IR3 mice, and at least 50-fold higher than in nontransgenic animals. RNA blot analysis of multiple tissues from IR10 mice demonstrated high level transgene expression in skin but not in other organs (Fig. 1). Prolonged exposure of this blot also demonstrated a weak signal in thymus RNA reflecting transgene expression in a subset of thymic epithelial cells (not shown). Analysis of thymocyte number and surface markers did not reveal a difference between IR10 and nontransgenic littermates. A second message of $\sim 2.5 \mathrm{~kb}$ was also observed in skin from the transgenic animals. Although the reason for this band is uncertain, it most likely represents use of noncanonical polyadenylation signals in the $3^{\prime}$ untranslated region of the IL-1 receptor cDNA.

To confirm appropriate cell surface expression of type 1 IL-1R protein, ear skin keratinocytes were isolated from FVB, IR3, and IR10 mice and analyzed by flow cytometry using 35F5, a monoclonal antibody which specifically recognizes the type 1 IL-1 receptor (Fig. 2). In nontransgenic animals no type 1 IL-1 receptor could be demonstrated by this technique. Low or absent expression was apparent in keratinocytes from IR3 mice, but readily observable cell surface expression of the type 1 IL-1R was detected in keratinocytes from IR10 animals. Additional experiments confirmed that expression of type 1 IL$1 \mathrm{R}$ was maintained by IR10 keratinocytes in culture (not shown). No type 2 IL-1R could be detected by flow cytometry on freshly isolated keratinocytes derived from either FVB mice or either transgenic line, and levels of IL-1R type 2 mRNA did not differ in FVB and IR10 mice (not shown).

The transgene-derived keratinocyte $I L-1 R$ is functional in vitro. Previous work from our laboratory and by others has shown that production of GM-CSF by keratinocytes in culture is critically dependent upon levels of extracellular IL-1 $(8,10)$. Therefore, to examine the functional consequence of overexpression of keratinocyte type 1 IL-1R in vitro, keratinocytes derived from IR10 mice and non transgenic littermates were cultured in varying concentrations of exogenously added IL$1 \alpha$, and GM-CSF secretion into the medium was assayed by ELISA. Keratinocytes were cultured under conditions that maintain expression of keratin 14, and therefore transgenes under control of the keratin 14 promoter (22). Fig. 3 shows the expected dose-response relationship between exogenous IL-1 and GM-CSF production in FVB mice. However, when IR10 keratinocytes were exposed to the same concentrations of IL$1 \alpha$, higher levels of GM-CSF production were observed, and in the experiment shown in Fig. 3, keratinocytes were maximally stimulated at the lowest level $(30 \mathrm{pg} / \mathrm{ml})$ of IL- $1 \alpha$ used. Fur-

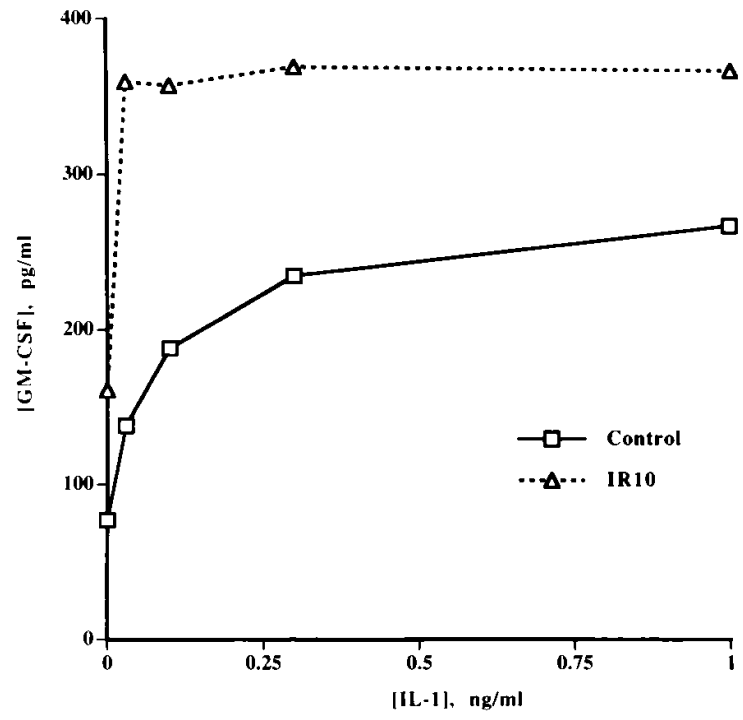

Figure 3. GM-CSF production by IR10 and control keratinocytes. Keratinocytes were cultured in varying concentrations of recombinant murine IL- $1 \alpha$ for $24 \mathrm{~h}$ and GM-CSF secreted into the supernatants was measured by ELISA. Data reflects the mean of triplicate cultures and is representative of three independent experiments. 
Table I. Ear Swelling Responses of IR10 and Nontransgenic FVB Animals to Application of PMA $0.5 \mu \mathrm{g}$ to Each Side of the Right Ear on Days 0, 3, and 6

\begin{tabular}{cccc}
\hline Day & FVB $(n=4)$ ear swelling & IR10 $(n=4)$ ear swelling & $P=$ \\
\hline & $\mu m$ & $\mu m$ & \\
0 & 0 & 0 & $\mathrm{~ns}$ \\
1 & $95 \pm 27$ & $60 \pm 7$ & $\mathrm{~ns}$ \\
7 & $127 \pm 6$ & $242 \pm 54$ & $<0.01$ \\
\hline
\end{tabular}

Ear thickness was measured with a spring loaded micrometer, and data represent the mean increase in ear thickness compared to measurements taken immediately before application of the first dose of PMA. Vehicle (ethanol) was applied to the left ear as control: no swelling was detectable in either group. Similar results were obtained in two additional experiments. thermore, the low constitutive level of GM-CSF produced in the absence of added IL-1 was greater in the transgenic mice than in controls. Taken together, these findings confirm that the IL-1 receptor overexpressed on the surface of IR10 keratinocytes confers marked hypersensitivity to IL-1 in vitro.

IR10 mice respond abnormally to IL-1-mediated elicited inflammation. Unperturbed skin of IR10 mice is histologically normal. IR10 mice did not differ macroscopically from their littermates. Histological examination of the skin, tongue and forestomach (all sites in which the keratin 14 promoter is active) was unremarkable (not shown). In particular, no evidence for spontaneous inflammation was detected. Furthermore, examination of other organs, including liver, kidney, thymus, spleen, lung, and heart showed no evidence of inflammatory or other change.

Because a growing body of evidence suggests that keratinocyte release of IL-1 plays an important role in the cutaneous
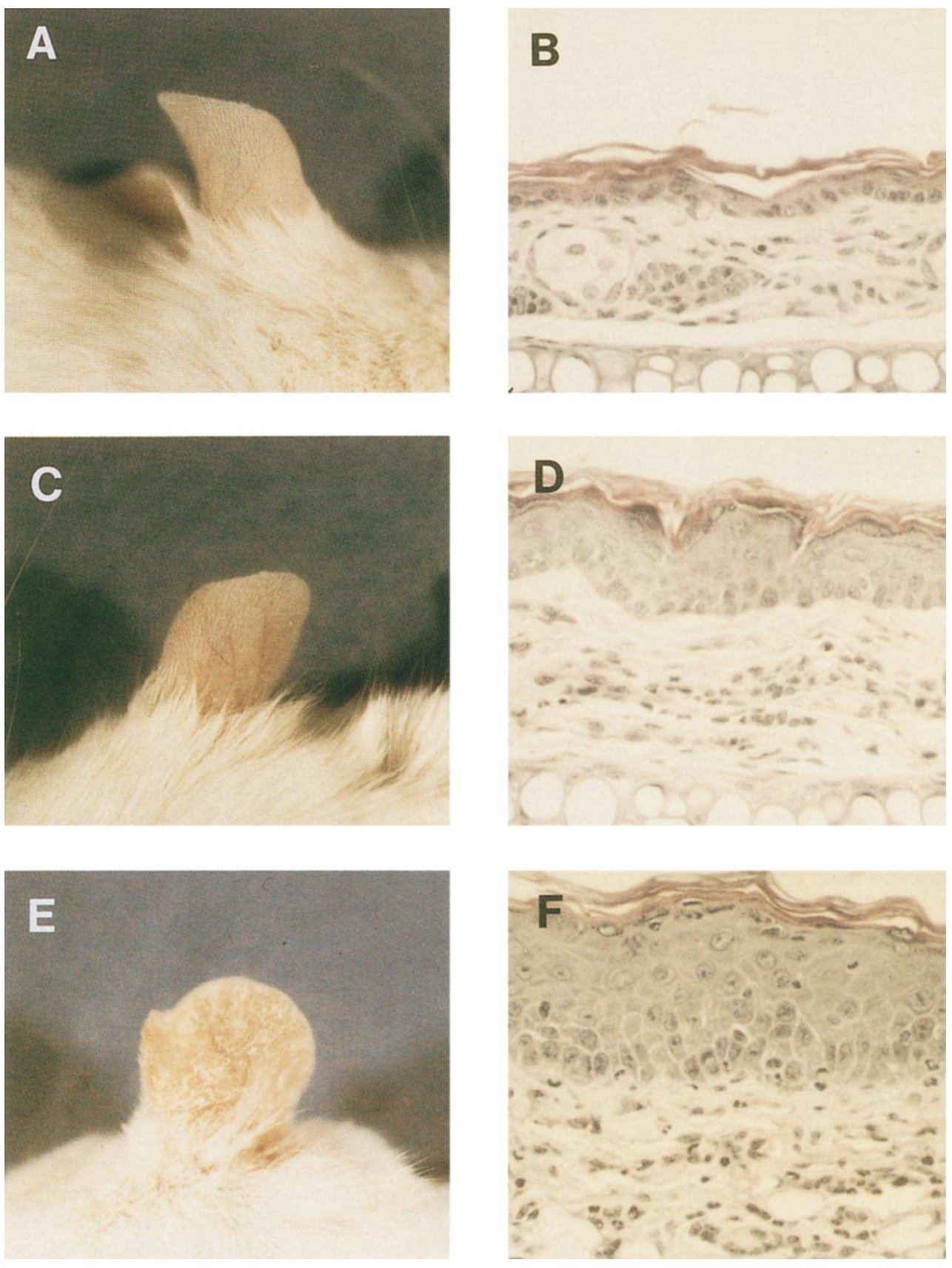

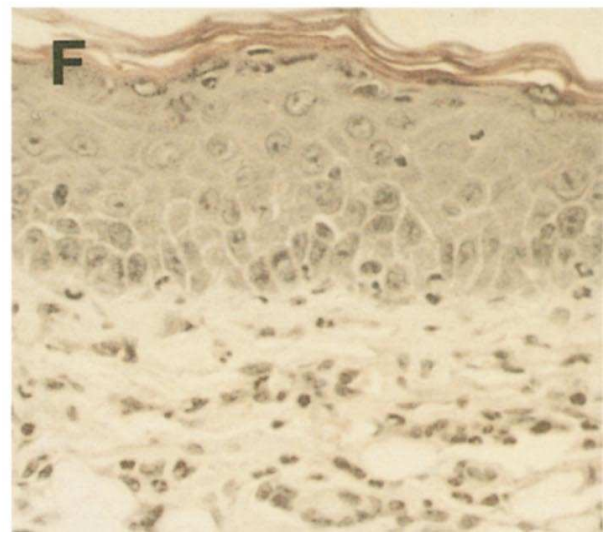

Figure 4. Phorbol ester treatment of nontransgenic and IR10 ears. PMA $(0.5 \mu \mathrm{g})$ was applied to each side of the right ear three times over one week as described. Vehicle (ethanol) alone was applied to the left ear. ( $A$ and $B$ ) Nontransgenic mouse, vehicle control. $(C$ and $D$ ) Nontransgenic mouse, PMA. ( $E$ and $F$ ) IR10 mouse, PMA. $(B, D$, and $F)$ Hematoxylin and eosin stain, $\times 100)$. 


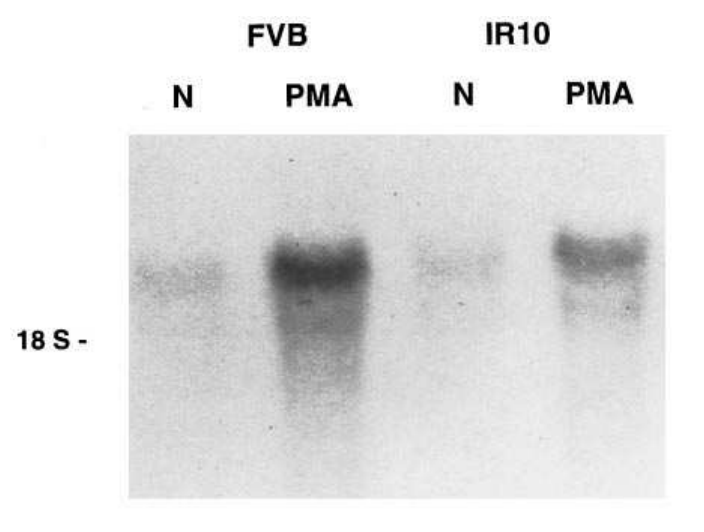

$18 \mathrm{~S}-$
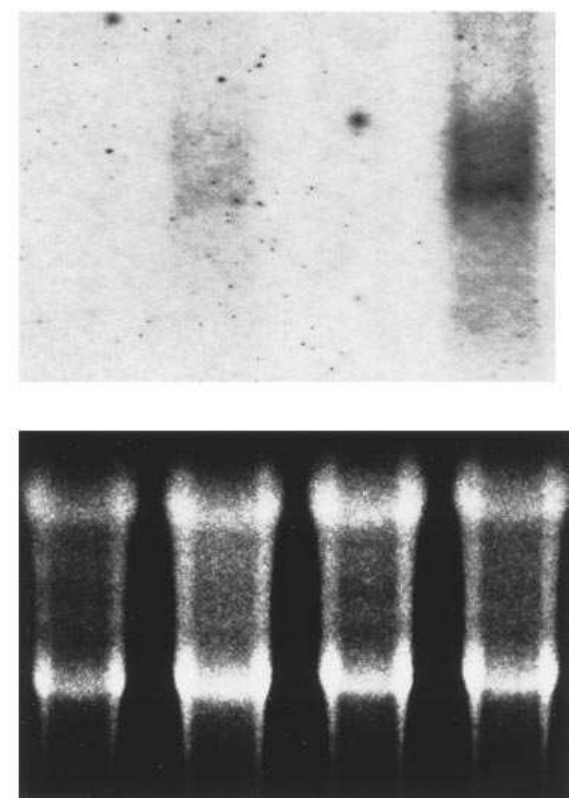

Figure 5. Cytokine induction in control and IR10 skin 4 hours following application of $1 \mu \mathrm{g}$ PMA. (Upper panel) IL-1 $\alpha$ mRNA is induced in both control and IR10 skin. (Middle panel) The same blot was stripped and reprobed for GM-CSF mRNA: this cytokine was strongly induced in IR10 skin. (Lower panel) ethidium bromide stained gel demonstrates equivalent RNA loading.

inflammatory response to topical application of phorbol 13myristate 12 -acetate $(32,33)$, we chose to examine the response of IR10 mice to this stimulus. Repeated application of PMA to ear skin resulted in a highly reproducible ear swelling response (Table I and Fig. 4); the magnitude of this swelling was significantly greater in transgenic mice than their littermate controls. Visual inspection of the ears of IR10 mice revealed that they were more markedly thickened, erythematous and scaly following PMA treatment compared to controls (Fig. 4). While in control animals this regimen of PMA application resulted in modest epidermal hyperplasia and minimal dermal inflammatory cell accumulation, in the IR10 mice these features were greatly exaggerated, with pronounced epidermal thickening, intraepithelial edema and a heavy dermal infiltrate of inflammatory cells (Fig. 4).

We hypothesized that these changes were related to excessive keratinocyte production of secondary cytokines in response to IL-1 stimulation. Therefore, we predicted that IL-1 inducible secondary cytokines would be overexpressed in the skin of the transgenic mice. To address this issue, we analyzed
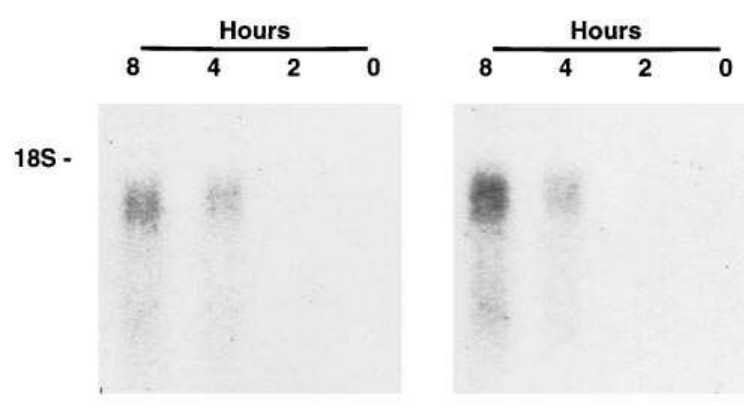

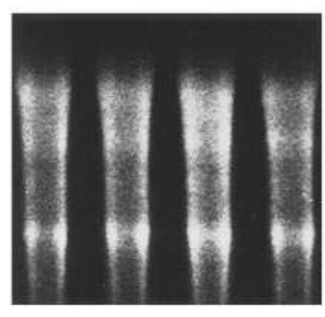

FVB
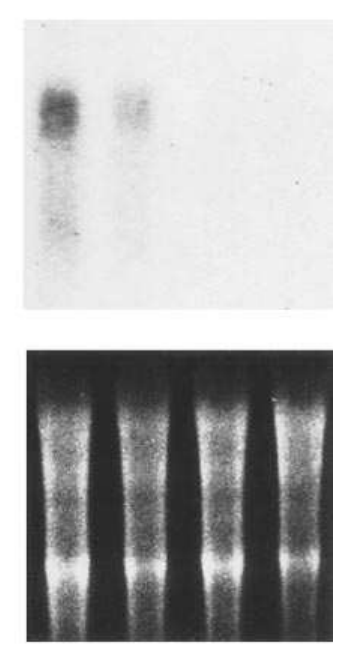

IR10
Figure 6. gro- $\alpha$ mRNA induction following PMA application to IR10 and nontransgenic skin. (Upper panel) PMA was applied to ears and RNA was extracted at the times indicated. Note exaggerated gro- $\alpha$ mRNA induction in IR10 ears. (Lower panel) Ethidium bromide stained gel demonstrates equivalent RNA loading.

RNA blots of PMA treated skin from control and transgenic mice at various times following application of PMA with labeled cytokine cDNA probes. Whereas IL- $1 \alpha$ mRNA was equivalently induced in control and transgenic epidermis (Fig. 5 , upper panel), the accumulation of mRNA for both GM-CSF (Fig. 5, middle panel) and the IL-1 inducible chemokine gro- $\alpha$ (Fig. 6) was significantly greater in skin of IR10 mice compared with their nontransgenic littermates. These findings are consistent with the interpretation that the changes observed in IR10 mice after PMA stimulation were related to excessive autocrine or paracrine stimulation of keratinocytes by IL-1.

$I L-1 / I L-1 R$ double transgenic mice have spontaneously inflamed skin. Although IL-1 has been implicated as a major effector molecule in the cutaneous response to epicutaneously applied PMA, we considered the possibility that other keratinocyte-derived proinflammatory cytokines (e.g., TNF $\alpha$ ) may also play a role. It was reasoned that by crossing IR10 mice to transgenic mice that overexpress IL- $1 \alpha$ in keratinocytes and examining animals doubly transgenic for both IL-1a and the type I IL-1R, we would be able to assess the effect of increased IL-1 agonist ligand receptor interactions in a completely unique fashion. Previous studies from our laboratory reported the generation of two lines of IL- $1 \alpha$ transgenic mice (21). A line exhibiting high level expression of IL-1 in basal keratinocytes (TgIL-1.1) exhibits generalized cutaneous inflammation; however, a line that expresses lower levels of IL- $1 \alpha$ (TgIL-1.2) does not exhibit spontaneous cutaneous inflammation before 6 months of age. We therefore crossed mice from the TgIL-1.2 line with IR10 mice and identified double transgenic animals in the offspring by PCR analysis of tail skin DNA. Double transgenic animals were immediately distinguishable from either single transgenic or nontransgenic littermates by virtue of their somewhat smaller size and delayed hair growth. Around the time of weaning, the skin of these an- 

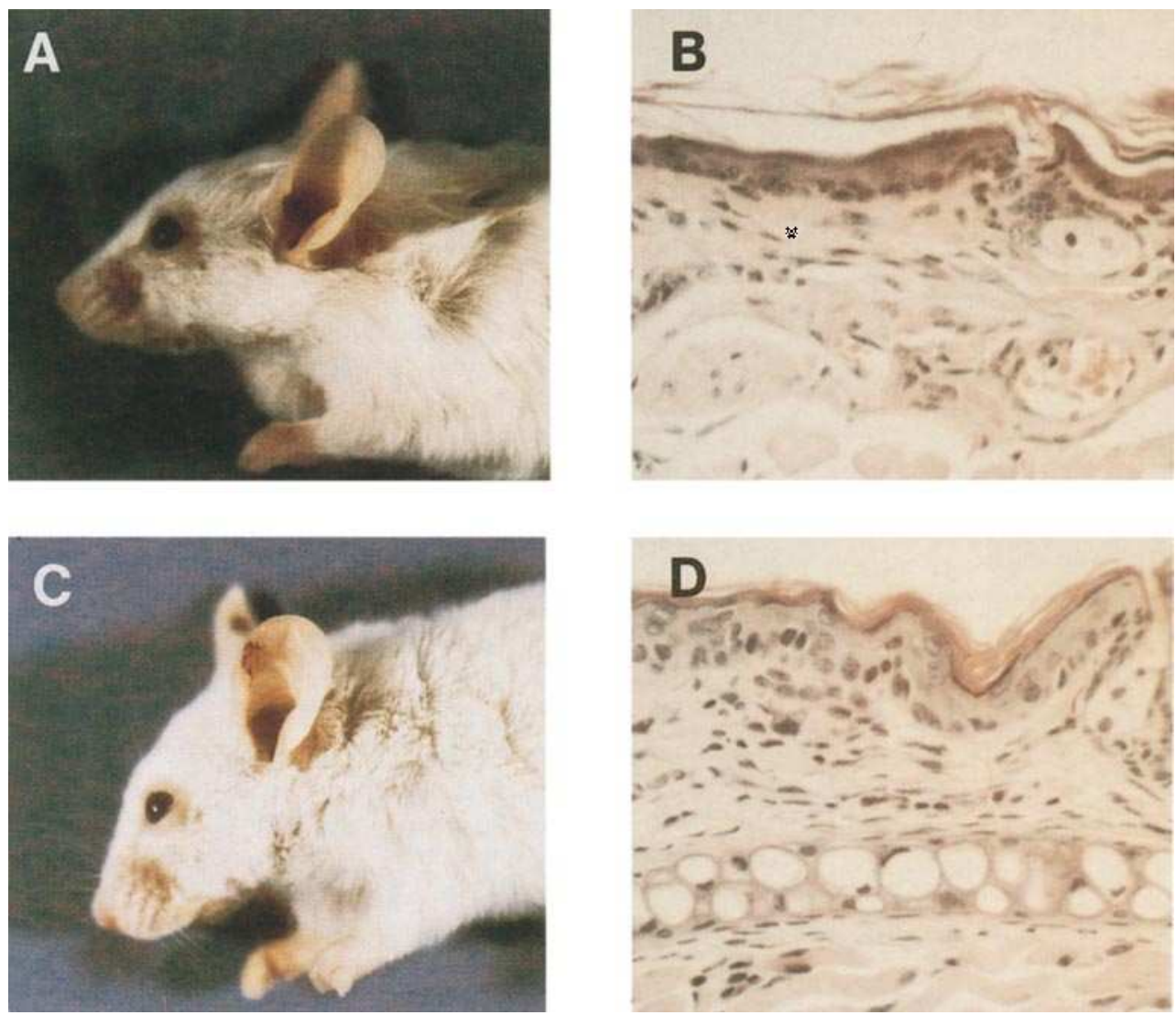

Figure 7. Spontaneous inflammatory changes in skin of mice which express both IL-1 and IL-1 receptor transgenes. ( $A$ and $B$ ) TgIL-1.2 mice have normal appearing ears. Histologically there is a slight increase in dermal mononuclear cells. $(C$ and $D$ ) TgIL-1.2 $\times$ IR10 double transgenic mice develop spontaneous erythema and thickening of the ears. Histologically this is characterized by epidermal hyperplasia and a dermal inflammatory infiltrate. ( $B$ and $D$ ) Hematoxylin and eosin stain, $\times 100$. imals was erythematous and exhibited a fine scale. As the animals matured, the skin of double transgenic mice developed spontaneous inflammatory changes (Fig. 7 C) and histological examination revealed a thickened epidermis with normal keratinization and a mixed dermal inflammatory infiltrate of macrophages, lymphocytes and some polymorphonuclear leukocytes (Fig. $7 \mathrm{D}$ ).

If the changes observed in the double transgenic mice were indeed related to keratinocyte activation by IL-1 mediated by the type 1 IL-1 receptor, we reasoned that secondary cytokine induction would occur to a greater degree in the double transgenic animals than either single transgenic line. We therefore examined the level of gro- $\alpha$ transcripts in skin of single and double transgenic mice (Fig. 8). As expected, little or no expression of gro- $\alpha$ was apparent in normal or IR10 skin. While only low levels of this mRNA were detected in TgIL-1.2 mice, gro- $\alpha$ mRNA was most strongly induced in the double transgenic animals, confirming the presence of IL-1 activation of keratinocytes in these animals.

\section{Discussion}

Autocrine activation of cells by cytokines has been suggested to play an important role in a wide variety of physiological processes (23), and the expression by a particular cell type of a soluble mediator and its receptor has generally been assumed to indicate the potential for an autocrine pathway of cell stimulation or activation. However, models that can test the potential for such autocrine pathways in vivo are difficult to design. In the present study, we have shown conclusively that keratinocytes expressing higher levels of the type I receptor respond more vigorously in vivo to keratinocyte IL-1 production by producing secondary cytokines. Moreover, this report demonstrates for the first time that such secondary cytokines produced by keratinocytes can amplify a cutaneous inflammatory response. This provides in vivo validation that keratinocytes, through both IL-1 production (21) and IL-1 inducible secondary cytokine production, may play a role in skin diseases.

The epidermal IL-1 system is highly complex (2), and because of the production of bioactive IL- $1 \alpha$ by resting keratinocytes in the context of constitutive presence of the signal transducing type 1 IL-1 receptor, we have suggested that IL-1-mediated autocrine pathways may exist within epidermis $(2,11)$. Furthermore, because of the pleiotropic inflammatory actions of IL-1, such pathways may be important in the pathogenesis of cutaneous inflammation, and alterations in levels of IL-1 (24, 25), IL-1ra (26) and IL-1 receptors (11) have been demonstrated in inflammatory skin disease. However, beyond the demonstration of the co-existence of biologically active IL-1 and type $1 \mathrm{IL}-1 \mathrm{R}$ in epidermis, in vivo confirmation of the importance of keratinocyte autocrine activation by IL-1 has been lacking.

Because of this potential importance of IL-1 in cutaneous physiology, we hypothesized that epidermis would be an important organ in which to study IL-1-mediated autocrine pathways. This hypothesis guided our decision to generate transgenic mice in which type 1 IL-1 receptor was targeted to basal keratinocytes under the control of a keratin-14-specific promoter/enhancer. We reasoned that by overexpressing the receptor we would be able to examine, in relative isolation, the consequences of excessive IL-1 feedback to keratinocytes. The IR10 line of transgenic mice expressed high levels of type 1 IL-1 
FVB

IR10 TgIL-1.2 $\underset{\text { XIR10 }}{\text { TgIL-1.2 }}$

$18 \mathrm{~S}-$
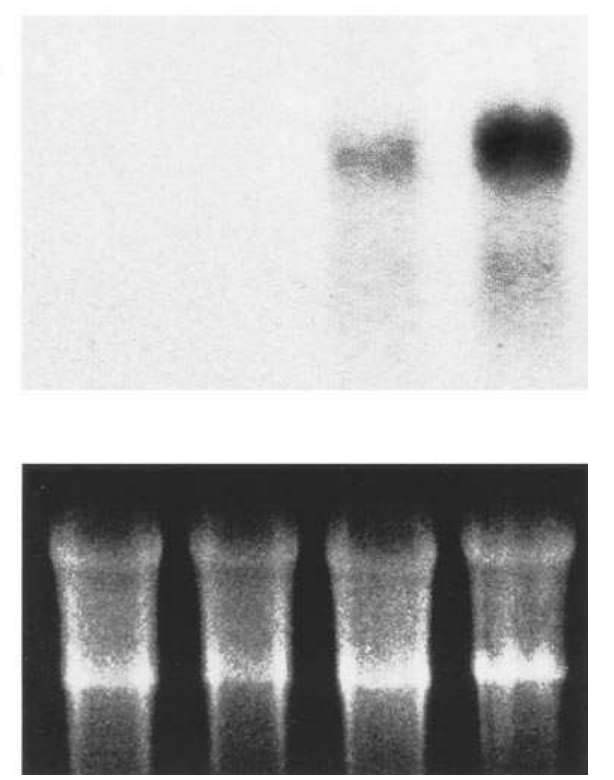

Figure 8. gro- $\alpha$ mRNA is overexpressed in TgIL-1.2 $\times$ IR10 double transgenic mice. RNA was extracted from littermates resulting from a TgIL-1.2 $\times$ IR10 mating. No gro- $\alpha$ mRNA is expressed in nontransgenic or IR10 skin. Low level constitutive expression is apparent in the TgIL-1.2 mice but there is marked induction in double transgenic animals.

receptor mRNA and protein. To determine whether this transgenic receptor was functional, keratinocytes were cultured under conditions that permit maintenance of keratin 14 (and transgene) expression; under such conditions, keratinocytes from these animals showed an exaggerated response to IL-1. The dose-response curve in these experiments was shifted to the left by over three orders of magnitude, such that transgenic keratinocytes were maximally stimulated at IL-1 concentrations that were almost invisible to normal keratinocytes. These findings confirm and extend previous in vitro transfection experiments (27) in which expression of type 1 IL-1R conferred IL-1 responsiveness to normally nonresponsive cells. Such experiments support the contention that these animals represent a valid model in which to study in vivo the consequences of keratinocyte overexpression of type 1 IL-1 receptor.

The lack of any secondary cytokine induction, gross or microscopic change in unperturbed skin of IR10 mice is of interest and implies that levels of extracellular IL-1 in FVB/N epidermis are very low or absent, in part because of the extreme sensitivity of transgenic keratinocytes to IL-1. As with other cell types, the majority of keratinocyte associated IL-1 is intracellular $(28,29)$, but cellular fractionation studies have demonstrated some IL-1 associated with the cell membrane (30). Our data would suggest that this putative membrane associated IL-1 (at least in resting epidermis) is unable to interact with the type $1 \mathrm{IL}-1 \mathrm{R}$ on adjacent keratinocytes, although it does not exclude a role for membrane IL-1 in keratinocyte interactions with other epidermal cells.

Although protein kinase $\mathrm{C}$ activation results in a variety of intracellular events, a considerable amount of data now suggests that the cutaneous inflammatory response to phorbol esters is largely mediated by IL-1. In vitro, PMA rapidly induces both synthesis and release of IL-1 by keratinocytes (31), and in vivo both early and late cutaneous responses to PMA are inhibited by administration of anti-IL-1 antibodies $(32,33)$. Furthermore, we have recently demonstrated that immediate vascular leakage in murine skin following topical PMA application can be largely abrogated by an antibody to the type-1 IL-1 receptor (T. Rauschmayr, R.W. Groves, and T.S. Kupper, manuscript submitted for publication). It was therefore of particular interest to examine the response of IR10 mice to this stimulus. By a number of criteria (macroscopic changes, ear swelling, epidermal hyperplasia and dermal inflammatory cell infiltrate), the response to a modest (submaximal) application of topical PMA was exaggerated in the IR10 mice. Furthermore, although IL-1 $\alpha$ mRNA was equally induced in control and IR10 animals, GM-CSF and gro- $\alpha$ mRNA's were more strongly induced in IR10 mice. Taken together, these findings strongly suggest that a significant component of the inflammatory response to phorbol esters in the skin is related to secondary cytokine production by keratinocytes, mediated by the type 1 IL-1 receptor, as these two groups differed only in their expression of the type 1 IL-1R.

To confirm that the changes seen in the IR10 mice truly represented an alteration in response to IL-1, and not some confounding variable also induced by PMA, we took advantage of a line of transgenic animals which constitutively overexpress (and release) IL- $1 \alpha$ in basal epidermis (21). We reasoned that this would enable us in a very specific fashion to examine the consequences of increased type 1 IL-1R expression in the context of excessive keratinocyte IL-1 release. Doubly transgenic animals developed spontaneous cutaneous inflammation similar in nature and degree to that elicited by PMA in the IR10 single transgenic mice. Macroscopic scaling, thickening and erythema of the skin were accompanied by histological evidence of epidermal hyperplasia and a dermal inflammatory cell infiltrate, changes not observed in either single transgenic line. The fact that these changes occurred maximally in the double transgenic mice indicates that a major part of this pathophysiologic process depends upon secondary keratinocyte responses to IL-1 rather than being a direct effect of IL-1 release. Consistent with this hypothesis is our observation that gro- $\alpha$ mRNA, while slightly induced in the TgIL-1.2 line, was markedly overexpressed in the double transgenic animals.

In our initial studies of IL- $1 \alpha$ transgenic mice we found that animals which expressed this transgene at very high levels developed a spontaneous inflammatory skin disease (21). However, the degree to which this was a result of direct IL-1 release by keratinocytes, systemic effects of IL-1, or secondary cytokine release could not be determined with certainty in this model. The present studies make clear that secondary cytokine release by keratinocytes can have a profound effect in such systems. Taken together, the findings presented in this paper provide direct evidence for an in vivo pathway of keratinocyte autocrine stimulation by IL-1. Stimulation of keratinocytes by PMA leads to both increased intracellular accumulation of IL-1 and its release into the extracellular space. Keratinocyte derived IL-1 can cross the epidermal basement membrane to activate cells in the dermis (e.g., fibroblasts and epithelial cells) as well as adjacent epidermal cells (e.g., keratinocytes, Langerhan's cells, and melanocytes). Such autocrine or juxtacrine keratinocyte stimulation by IL-1 may induce a plethora of secondary responses including the induction of secondary cytokines, 
proteases and increased keratinocyte mobility. We have previously termed such cells "activated keratinocytes" $(1,2)$.

Our findings in vivo underscore the importance of tight control of keratinocyte type $1 \mathrm{IL}-1$ receptor expression in vivo. This receptor functions as an integrator of extracellular IL-1 agonist and antagonist activities and, because occupation of very few receptors is required for cell activation $(6,27)$, small changes in the levels of IL-1 receptor expression can result in a significant decrease in the net extracellular IL-1 activity required for cell activation. In accordance with this, studies of the promoter region of type 1 IL-1R show similarities to many housekeeping genes suggesting that (at least at a transcriptional level) expression of this receptor is relatively constant (34). Clearly, in view of the numerous actions of IL-1 it makes good biological sense that expression of its signal transducing receptor is maintained at a constant basal level.

These studies emphasize the importance for regulated expression of different members of an interacting cytokine network. Such a system has been demonstrated in the ovarian microenvironment (35), and our data make it clear that within the epidermis, dysregulation of one part of the IL-1 system can lead to important alterations in the tissue response to external stimuli. Several studies have demonstrated abnormalities of IL-1 receptor expression in cutaneous inflammatory disease $(11,36)$ and our data indicate that dysregulated IL-1 receptor expression could play a primary role in such processes. Finally, the role of the type 1 IL-1 receptor as an integrator of extracellular IL-1 agonist and antagonistic activity may make it an attractive target for therapeutic intervention.

\section{Acknowledgments}

This work was supported by National Institutes of Health grants AR40124, AI-25082, and AR-42689 (to T.S. Kupper), and by the Harvard Skin Disease Research Center at Brigham and Women's Hospital. R.W. Groves gratefully acknowledges the support of the Wellcome Trust, the British Association of Dermatologists, The Skin Disease Research Fund and the Dermatology Foundation.

\section{References}

1. Kupper, T.S. 1990. Immune and inflammatory processes in cutaneous tissues: mechanisms and speculations. J. Clin. Invest. 86:1783-1789.

2. Kupper, T.S., and R.W. Groves. 1995. The Interleukin-1 axis and cutaneous inflammation. J. Invest. Dermatol. 105:62S-66S.

3. Gahring, L.C., A. Buckley, and R.A. Daynes. 1985. Presence of epidermal-derived thymocyte activating factor/interleukin 1 in normal human stratum corneum. J. Clin. Invest. 76:1585-1591.

4. Hauser, C., J-H. Saurat, A. Schmitt, F. Jaunin, and J-M. Dayer. 1986. Interleukin 1 is present in normal human epidermis. J. Immunol. 136:3317-3321.

5. Mizutani, H., R. Black, and T.S. Kupper. 1991. Human keratinocytes produce but do not process pro-interleukin-1 (IL-1) beta. Different strategies of IL-1 production and processing in monocytes and keratinocytes. J. Clin. Invest. 87:1966-1971.

6. Dower, S.K., J.E. Sims, D.P. Cerretti, and T. Bird. 1992. The interleukin-1 system: receptors, ligands and signals. Chem. Immunol. 51:33-64.

7. Dinarello, C.A. 1988. Biology of interleukin 1. FASEB J. 2:108-115.

8. Kupper, T.S., F. Lee, N. Birchall, S. Clark, and S.K. Dower. 1988. Interleukin 1 binds to specific receptors on human keratinocytes and induces granulocyte macrophage colony-stimulating factor mRNA and protein. J. Clin. Invest. $82: 1787-1792$

9. Sims, J.E., B. Acres, C.E. Grubin, C. McMahan, J.M. Wignall, C.J. March, and S.K. Dower. 1989. Cloning the IL-1 receptor from human T cells. Proc. Natl. Acad. Sci. USA. 86:8946-8950.

10. Groves, R.W., J.G. Giri, J.E. Sims, S.K. Dower, and T.S. Kupper. 1995. Inducible expression of type 2 IL-1 receptors by cultured human keratinocytes: implications for IL-1 mediated processes in epidermis. J. Immunol. 154:40654072.
11. Groves, R.W., L. Sherman, H. Mizutani, S.K. Dower, and T.S. Kupper. 1994. Detection of IL-1 receptors in human epidermis. Induction of the type 2 receptor after organ culture and in psoriasis. Am. J. Pathol. 145:1048-1056.

12. Deyerle, K.L., J. E. Sims, S.K. Dower, and M.A. Bothwell. 1992. Pattern of IL-1 receptor gene expression suggests role in noninflammatory processes. $J$. Immunol. 149:1657-1665.

13. Colotta, F., F. Re, M. Muzio, R. Bertini, N. Polentarutti, M. Sironi, J.G. Giri, S. K. Dower, J.E. Sims, and A. Mantovani. 1993. Interleukin-1 type II receptor: a decoy target for IL-1 that is regulated by IL-4. Science (Wash. DC). 261:472-475.

14. Re, F., M. Muzio, M. de Rossi, N. Polentarutti, J.G. Giri, A. Mantovani, and F. Colotta. 1994. The type II "receptor" as a decoy target for interleukin 1 in polymorphonuclear leukocytes: characterization of induction by dexamethasone and ligand binding properties of the released decoy receptor. J. Exp. Med. 179:739-743.

15. Haskill, S., G. Matin, L. Van Le, J. Morris, A. Peace, C.F. Bigler, G.J. Jaffe, C. Hammerberg, S.A. Spron, S. Fong, W.P. Arend, and P. Ralph. 1991. cDNA cloning of an intracellular form of the human interleukin 1 receptor antagonist associated with epithelium. Proc. Natl. Acad. Sci. USA. 88:3681-3685.

16. Eisenberg, S.P., M.T. Brewer, E. Verderber, P.L. Heimdal, B.J. Brandhuber, and R.C. Thompson. 1991. Interleukin 1 receptor antagonist is a member of the interleukin 1 gene family: Evolution of a cytokine control mechanism. Proc. Natl. Acad. Sci. USA. 88:5232-5236.

17. Bigler, C.F., D.A. Norris, W.L. Weston, and W.P. Arend. 1992. Interleukin-1 receptor antagonist production by human keratinocytes. J. Invest. Dermatol. 98:38-44.

18. Muzio, M., N. Polentarutti, M. Sironi, G. Poli, L. De Gioia, M. Introna, A. Mantovani, and F. Colotta. 1995. Cloning and characterization of a new isoform of the interleukin 1 receptor antagonist. J. Exp. Med. 182:623-628.

19. Sims, J.E., C. J. March, D. Cosman, M.B. Widmer, H.R. MacDonald, C. McMahan, C.E. Grubin, J.M. Wignall, J. Jackson, S.M. Call, D. Friend, A. Alpert, S. Gillis, D.L. Urdal, and S.K. Dower. 1988. cDNA expression cloning of the IL-1 receptor, a member of the immunoglobulin superfamily. Science (Wash. DC). 241:585-589.

20. Vassar, R., and E. Fuchs. 1991. Transgenic mice provide new insights into the role of TGF-alpha during epidermal development and differentiation. Genes. Dev. 5:714-727.

21. Groves, R.W., H. Mizutani, J.D. Kieffer, and T.S. Kupper. 1995. Inflammatory skin disease in transgenic mice that express high levels of interleukin 1a in basal epidermis. Proc. Natl. Acad. Sci. USA. 92:11874-11878.

22. Williams, I.R., and T.S. Kupper. 1994. Epidermal expression of intercellular adhesion molecule 1 is not a primary inducer of cutaneous inflammation in transgenic mice. Proc. Natl. Acad. Sci. USA. 91:9710-9714.

23. Sporn, M.B., and A.B. Roberts. 1992. Autocrine secretion-10 years later. Ann. Intern. Med. 117:408-414.

24. Cooper, K.D., C. Hammerberg, O. Baadsgaard, J.T. Elder, L.S. Chan, D.N. Sauder, J.J. Voorhees, and G. Fisher. 1990. IL-1 activity is reduced in psoriatic skin. Decreased IL-1 alpha and increased nonfunctional IL-1 beta. J. Immunol. 144:4593-4603.

25. Gearing, A.J.H., N.J. Fincham, C.R. Bird, M. Wadhwa, A. Meager, J.E. Cartwright, and R.D.R. Camp. 1990. Cytokines in skin lesions of psoriasis. $C y$ tokine. 2:68-75.

26. Hammerberg, C., W.P. Arend, G. Fisher, L.S. Chan, A.E. Berger, J.S. Haskill, J.J. Voorhees, and K.D. Cooper. 1992. Interleukin-1 receptor antagonist in normal and psoriatic epidermis. J. Clin. Invest. 90:571-583.

27. Curtis, B.M., B. Gallis, R.W. Overell, C. McMahan, P. DeRoos, R. Ireland, J. Eisenman, S.K. Dower, and J.E. Sims. 1989. T-cell interleukin 1 receptor cDNA in Chinese hamster ovary cells regulates functional responses to interleukin 1. Proc. Natl. Acad. Sci. USA. 86:3045-3049.

28. McGuire, J., L. Didierjean, R.C. Langdon, J-M. Dayer, and J-H. Saurat. 1988. The subcellular distribution of different molecular weight forms of interleukin-1 alpha and beta in the human keratinocyte. J. Invest. Dermatol. 90:587593.

29. Anttila, H.S.I., S. Reitamo, P. Erkko, A. Miettinen, L. Didierjean, and J-H. Saurat. 1990. Membrane and cytosolic interleukin-1 alpha and beta in normal human epidermal cells: Variability of epitope exposure in immunohistochemistry. J. Invest. Dermatol. 95:31-38.

30. Goldminz, D., T.S. Kupper, and J. McGuire. 1987. Keratinocyte membrane-associated epidermal cell-derived thymocyte-activating factor (ETAF). J. Invest. Dermatol. 88:97-100.

31. Blanton, R.A., T.S. Kupper, J.K. McDougall, and S.K. Dower. 1989. Regulation of interleukin 1 and its receptor in human keratinocytes. Proc. Natl. Acad. Sci. USA. 86:1273-1277.

32. Oberyszyn, T.M., C.L. K Sabourin, G.N. Bijur, A.S. Oberyszyn, L.G. Boros, and F.M. Robertson. 1993. Interleukin-1 $\alpha$ gene expression and localization of interleukin-1aprotein during tumor promotion. Mol. Carcinog. 7:238248.

33. Lee, W.Y., M.F. Locniskar, and S.M. Fischer. 1994. Interleukin-1 $\alpha$ mediates phorbol ester-induced inflammation and epidermal hyperplasia. FASEB J. 8:1081-1085.

34. Ye, K., C.A. Dinarello, and B.D. Clark. 1993. Identification of the promotor region of human interleukin-1 type-1 receptor gene: multiple initiation 
sites, high G + C content, and constitutive expression. Proc. Natl. Acad. Sci. USA. 90:2295-2299.

35. Simon, C., A. Frances, G. Piquette, and M.L. Polan. 1994. Immunohistochemical localization of the interleukin-1 system in the mouse ovary during follicular growth, ovulation, and luteinization. Biol. Reprod. 50:449-457.
36. Kristensen, M., B. Deleuran, D.J. Eedy, M. Feldmann, S.M. Breathnach, and F.M. Brennan. 1992. Distribution of interleukin 1 receptor antagonis protein (IRAP), interleukin 1 receptor, and interleukin 1 alpha in normal and psoriatic skin. Decreased expression of IRAP in psoriatic lesional epidermis. Br. J. Dermatol. 127:305-311. 